\title{
UNCORRELATED ENERGY SPREAD AND LONGITUDINAL EMITTANCE OF A PHOTOINJECTOR BEAM*
}

\author{
Z. Huang ${ }^{\dagger}$, D. Dowell, P. Emma, C. Limborg-Deprey, G. Stupakov, J. Wu \\ SLAC, Stanford, CA 94309, USA
}

\begin{abstract}
Longitudinal phase space properties of a photoinjector beam are important in many areas of high-brightness beam applications such as bunch compression, transverseto-longitudinal emittance exchange, and high-gain freeelectron lasers. In this paper, we discuss both the rf and the space charge contributions to the uncorrelated energy spread of the beam generated from a laser-driven rf gun. We compare analytical expressions for the uncorrelated energy spread and the longitudinal emittance with numerical simulations and recent experimental results.
\end{abstract}

\section{INTRODUCTION}

High-brightness electron beams generated by laserdriven rf guns have important applications in $\mathrm{x}$-ray free electron lasers (FEL) and advanced accelerator and light source R\&D. While the transverse phase space properties of these beams have been studied extensively, the uncorrelated energy spread and the longitudinal emittance have only been characterized recently at a few rf gun facilities [1, 2, 3]. Unlike the common desire to minimize the transverse emittance, the measured uncorrelated energy spread of a photoinjector beam is in fact too small to suppress a microbunching instability associated with acceleration and magnetic compression designed for shortwavelength FELs [4, 5]. To this end, a laser-based beam heater that increases the uncorrelated energy spread is designed in the Linac Coherent Light Source (LCLS) to control the beam quality degradation due to the instability [6]. Nevertheless, some other beam manipulation schemes such as transverse-to-longitudinal emittance exchange [7] and variable-wavelength high-gain harmonic generation FEL [8] require very small uncorrelated energy spread and longitudinal emittance. Therefore, a general understanding of these longitudinal beam parameters will be useful for a variety of high-brightness beam applications.

In this paper, we study the origins of the uncorrelated energy spread in an rf gun, taking into account both the rf and the space charge effects. We show that the main contribution to the uncorrelated energy spread comes from transverse variations of the longitudinal space charge field experienced by an intense electron bunch. We also discuss the longitudinal emittance and compare analytical estimations with simulations and experiments.

\footnotetext{
* Work supported by US Department of Energy contract DE-AC0276 SF00515.

† Email: zrh@slac.stanford.edu
}

\section{RF CONTRIBUTION TO THE UNCORRELATED ENERGY SPREAD}

It is known that the energy gain for an ultra-relativistic particle through an axially symmetric accelerating cavity is independent of the transverse offset of the particle. Nevertheless, in an rf gun electrons are accelerated from rest to relativistic velocities starting in the middle of a full-cell cavity. Thus, the energy gain due to the rf acceleration can depend on the transverse offset of the electron and may give rise to the uncorrelated energy spread of the beam.

To quantify the rf contribution, we use the paraxial representation of the $\mathrm{rf}$ accelerating field near the cavity axis (see, e.g., Ref. [9]). In MKS units, the longitudinal field is

$$
E_{z}(r, z, t) \approx\left[1-\frac{r^{2}}{4}\left(\frac{\partial^{2}}{\partial z^{2}}-\frac{1}{c^{2}} \frac{\partial^{2}}{\partial t^{2}}\right)\right] E_{z}(0, z, t)
$$

where $r=\sqrt{x^{2}+y^{2}}$ is the radial offset of the electron, and $z$ is the longitudinal distance from the cathode. The radial electric field and the azimuthal magnetic field are given by

$$
E_{r} \approx-\frac{r}{2} \frac{\partial E_{z}(0, z, t)}{\partial z}, \quad B_{\theta}=\frac{r}{2 c^{2}} \frac{\partial E_{z}(0, z, t)}{\partial t} .
$$

The electron motion is governed by the Lorentz force

$$
\frac{d(\gamma m \mathbf{v})}{d t}=e(\mathbf{E}+\mathbf{v} \times \mathbf{B})
$$

where $\mathbf{v}=(d r / d t, d z / d t)$ is the velocity vector. The energy gain can be calculated from

$$
\frac{d\left(\gamma m c^{2}\right)}{d t}=e \mathbf{v} \cdot \mathbf{E}
$$

We take the on-axis longitudinal field of the form

$$
E_{z}(0, z, t)=E_{0} \bar{E}(z) \sin \left(2 \pi f_{0} t+\phi_{0}\right)
$$

where $f_{0}$ is the $\mathrm{rf}$ frequency, $E_{0}$ is the maximum $\mathrm{rf}$ field amplitude, $\phi_{0}$ is the launch phase of the laser pulse relative to the rf zero-crossing, and $\bar{E}(z)$ is the dimensionless longitudinal field profile of a specific rf gun design. Equations (3) and (4) can be integrated numerically for a given $\bar{E}(z)$. For instance, the LCLS injector uses an Sband $11 / 2$-cell gun with $f_{0}=2856 \mathrm{MHz}$ and $\bar{E}(z)$ given by Omega3P simulations [10] as shown in Fig. 1. The on-axis electron energy $\gamma m c^{2}$ and the energy deviation $\Delta \gamma m c^{2}$ of an off-axis electron that leaves the cathode at $r=1 \mathrm{~mm}$ are shown in Fig. 2, for $E_{0}=120 \mathrm{MV} / \mathrm{m}$ and $\phi_{0}=30^{\circ}$. 


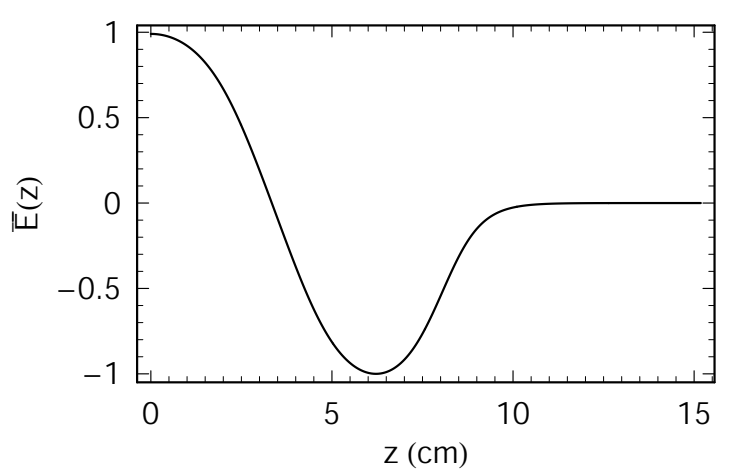

Figure 1: Dimensionless longitudinal field $\bar{E}(z)$ as a function of distance $z$ from the cathode in the LCLS gun.

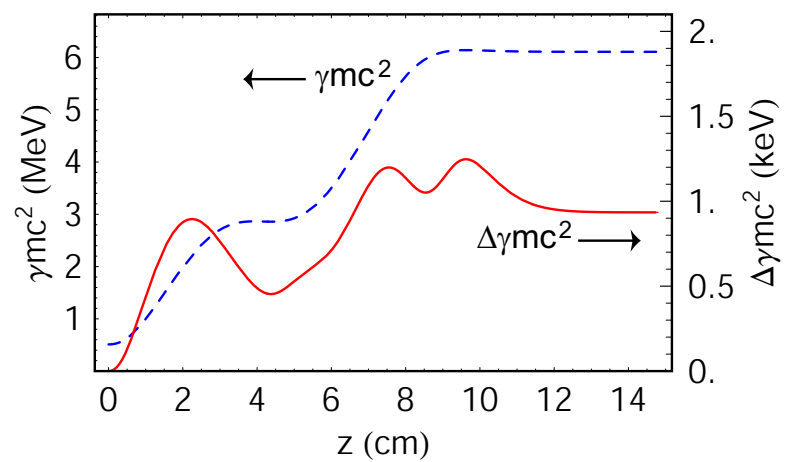

Figure 2: On-axis electron energy $\gamma m c^{2}$ (dashed blue curve) and energy deviation $\Delta \gamma m c^{2}$ for an off-axis electron that leaves the cathode at $r=1 \mathrm{~mm}$ (solid red curve).

The rf induced uncorrelated energy spread for an electron beam with the transverse size about $1 \mathrm{~mm}$ remains small $(<1 \mathrm{keV})$ for a wide range of the initial phase $\phi_{0}$ that produces a rapid acceleration in the gun. We will therefore neglect its contribution in comparison with the space charge effects discussed below.

\section{SPACE CHARGE CONTRIBUTION TO THE UNCORRELATED ENERGY SPREAD}

Space charge is the main collective effect in the rf gun. The transverse variation of the longitudinal space charge field can give rise to the uncorrelated energy spread. To estimate this contribution, we follow Kim [11] who observes that due to the typical $1 / \gamma^{2}$-dependence of the space charge forces, the dominant space charge contribution in the gun comes from the region where $\gamma \approx 1$. In this approximation, the total space charge induced energy change is [11]

$$
\Delta \gamma(r, s) \approx \frac{\pi}{2} \frac{m c^{2}}{e E_{0} \sin \phi_{0}} \frac{I_{0}}{I_{A}} \mathcal{E}_{z}(r, s)
$$

where $I_{0}$ is the peak current, $I_{A} \approx 17 \mathrm{kA}$ is the Alfvén current, $s$ is the longitudinal coordinate relative to the bunch center (with the bunch head at $s<0$ ), and the normalized

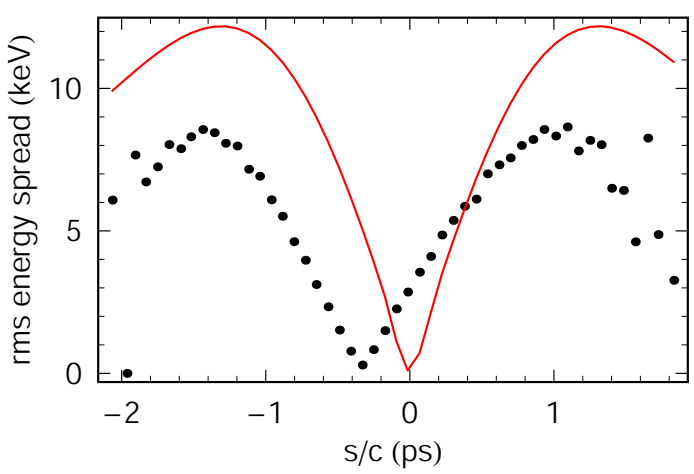

Figure 3: Uncorrelated rms energy spread as a function of bunch position $s / c$ (head at $s<0$ ) from Parmela (black symbol) and from Eq. (8) (red curve).

longitudinal space charge field (at $\gamma=1$ ) is

$$
\mathcal{E}_{z}(r, s)=\frac{s}{\sigma_{s}^{2}} \int_{0}^{\infty} d \xi \frac{\exp \left[-\frac{r^{2} A^{2}}{2 \sigma_{x}^{2}\left(A^{2}+\xi\right)}+\frac{s^{2}}{2 \sigma_{s}^{2}(1+\xi)}\right]}{(1+\xi)^{3 / 2}\left(A^{2}+\xi\right)}
$$

for a three-dimensional Gaussian charge distribution with a transverse rms size $\sigma_{x}=\sigma_{y}$ and an rms bunch length $\sigma_{s}=\sigma_{x} / A$. As expected, the longitudinal space charge field at any bunch position $s$ depends on its transverse offset $r$. Taking rms over the transverse Gaussian distribution, the space charge induced uncorrelated energy spread as a function of the longitudinal bunch coordinate $s$ is

$$
\begin{aligned}
\sigma_{\gamma}(s) & =\sqrt{\left\langle\Delta \gamma^{2}\right\rangle_{r}-\langle\Delta \gamma\rangle_{r}^{2}} \\
& \approx \frac{\pi}{2} \frac{m c^{2}}{e E_{0}\left(\sin \phi_{0}\right) \sigma_{s}} \frac{I_{0}}{I_{A}} F\left(\frac{s}{\sigma_{s}}\right),
\end{aligned}
$$

where

$$
\begin{aligned}
F^{2}(u)= & u^{2} \int_{0}^{\infty} \frac{d \xi_{1} e^{-u^{2} /\left[2\left(1+\xi_{1}\right)\right]}}{\left(1+\xi_{1}\right)^{3 / 2}} \int_{0}^{\infty} \frac{d \xi_{2} e^{-u^{2} /\left[2\left(1+\xi_{2}\right)\right]}}{\left(1+\xi_{2}\right)^{3 / 2}} \\
& \times \frac{1}{3 A^{4}+2 A^{2}\left(\xi_{1}+\xi_{2}\right)+\xi_{1} \xi_{2}} \\
& -\left[\int_{0}^{\infty} d \xi \frac{e^{-u^{2} /[2(1+\xi)]}}{(1+\xi)^{3 / 2}\left(2 A^{2}+\xi\right)}\right]^{2}
\end{aligned}
$$

Let us compare Eq. (8) with the uncorrelated energy spread obtained from the Parmela [12] space charge simulation of the Gun Test Facility (GTF) at SLAC with the following parameters: $E_{0}=110 \mathrm{MV} / \mathrm{m}, \phi_{0}=30^{\circ}$, $I_{0}=130 \mathrm{~A}$, and a Gaussian laser pulse with $\sigma_{s}=0.27$ $\mathrm{mm}(0.9 \mathrm{ps})$. We approximate the uniform transverse laser pulse with $1-\mathrm{mm}$ radius by a Gaussian shape with $\sigma_{x}=1 / \sqrt{3} \approx 0.58 \mathrm{~mm}$ to obtain the bunch aspect ratio $A=\sigma_{x} / \sigma_{s} \approx 2$. As shown in Fig. 3, the uncorrelated energy spread predicted from Eq. (8) agrees reasonably well with the simulation results obtained at the exit of the gun, indicating the transverse variation of the longitudinal space charge field provides the dominant contribution. Parmela simulations also show that the uncorrelated energy spread does not increase much after the rf gun (see, e.g., Fig. 8 of Ref. [13]). 
The uncorrelated energy spread varies along the bunch length. For convenience, we may define an average uncorrelated energy spread as

$$
\begin{aligned}
\left(\bar{\sigma}_{\gamma}\right)_{\mathrm{unc}} & \equiv\left[\int_{-\infty}^{\infty} \frac{d s}{\sqrt{2 \pi} \sigma_{s}} \sigma_{\gamma}^{2}(s) \exp \left(-\frac{s^{2}}{2 \sigma_{s}^{2}}\right)\right]^{1 / 2} \\
& =\frac{\pi}{2} \frac{m c^{2}}{e E_{0}\left(\sin \phi_{0}\right) \sigma_{s}} \frac{I_{0}}{I_{A}} G(A)
\end{aligned}
$$

where

$$
\begin{aligned}
G^{2}(A)= & A^{4} \int_{0}^{\infty} d \xi_{1} \int_{0}^{\infty} \frac{d \xi_{2}}{\left(3+2 \xi_{1}+2 \xi_{2}+\xi_{1} \xi_{2}\right)^{3 / 2}} \\
& \times \frac{1}{4 A^{4}+2 A^{2}\left(\xi_{1}+\xi_{2}\right)+\xi_{1} \xi_{2}} \\
& \times \frac{1}{3 A^{4}+2 A^{2}\left(\xi_{1}+\xi_{2}\right)+\xi_{1} \xi_{2}}
\end{aligned}
$$

Equation (10) yields $\left(\bar{\sigma}_{\gamma}\right)_{\mathrm{unc}} \approx 9 \mathrm{keV}$ for the above GTF parameters. For the LCLS gun, we use $E_{0}=120 \mathrm{MV} / \mathrm{m}$, $\phi_{0}=30^{\circ}, I_{0}=100 \mathrm{~A}, \sigma_{x}=0.57 \mathrm{~mm}$, and a Gaussian laser pulse with $\sigma_{s}=0.87 \mathrm{~mm}(2.9 \mathrm{ps})$. Then the average uncorrelated energy spread is $\left(\bar{\sigma}_{\gamma}\right)_{\text {unc }} \approx 5 \mathrm{keV}$. Parmela simulations of the LCLS injector using a flattop laser pulse with the same rms bunch length (about 10 ps fwhm) show $3 \mathrm{keV}$ rms uncorrelated energy spread, which is consistent with this estimation since the longitudinal space charge effects are somewhat reduced for a flattop current profile. Finally, the uncorrelated energy spread is measured at the TTF photoinjector [1] with the following parameters: $E_{0}=$ $40 \mathrm{MeV}, \phi_{0}=40^{\circ}, \sigma_{x}=3 / \sqrt{3} \approx 1.7 \mathrm{~mm}, \sigma_{s} \approx 2 \mathrm{~mm}$

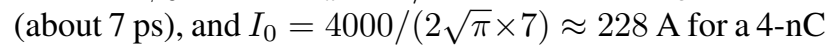
bunch. From Eq. (10), we obtain $\left(\bar{\sigma}_{\gamma}\right)_{\text {unc }} \approx 10 \mathrm{keV}$, which is about twice the measured average (close to $5 \mathrm{keV}$ ) [1].

\section{LONGITUDINAL EMITTANCE}

The rms longitudinal emittance characterizes the longitudinal phase space area of the beam enclosed by a simple ellipse. For a typical short electron bunch, the nonlinear $s$-dependent rf effect is negligible, and the rms longitudinal emittance is determined by both the $r$-dependent and the nonlinear $s$-dependent space charge effects in the gun, given approximately by [11]

$$
\epsilon_{s} \approx \frac{\pi}{2} \frac{m c^{2}}{e E_{0} \sin \phi_{0}} \frac{I_{0}}{I_{A}} \frac{1.1}{\left(1+4.5 A+2.9 A^{2}\right)} .
$$

Since the main effects occur in the region where $\gamma \approx 1$, we take the initial rms bunch length $\sigma_{s}$ (given by the laser pulse length) in calculating $I_{0}$ and $A$ even though the measured final rms bunch length $\left(\sigma_{s}\right)_{f}$ varies with the bunch charge. Figure 4 shows the longitudinal emittance measured in the GTF injector [2] for various bunch charges, in comparison with Eq. (12) using parameters discussed in the above section. We can also define an effective uncorrelated energy spread by $\left(\sigma_{\gamma}\right)_{\text {eff }}=\epsilon_{s} /\left(\sigma_{s}\right)_{f}$ and compare the theoretical expectations with the measured data [2] in Fig. (5). In both

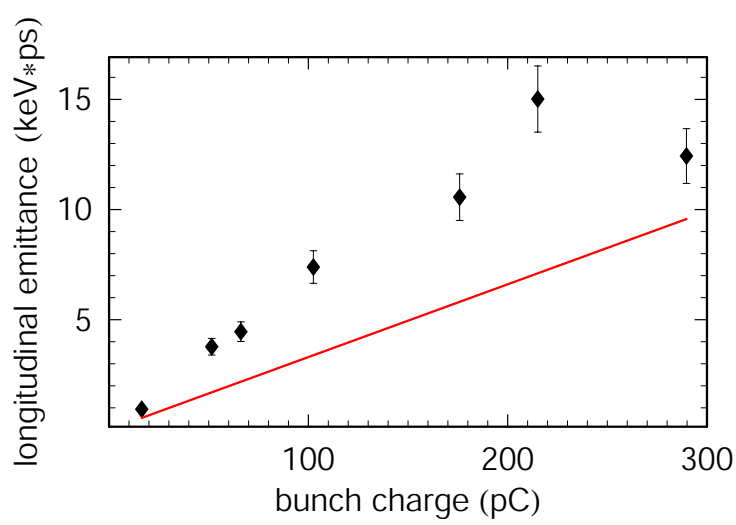

Figure 4: Longitudinal emittance measured in the GTF (black symbol) and obtained from Eq. (12) (red curve).

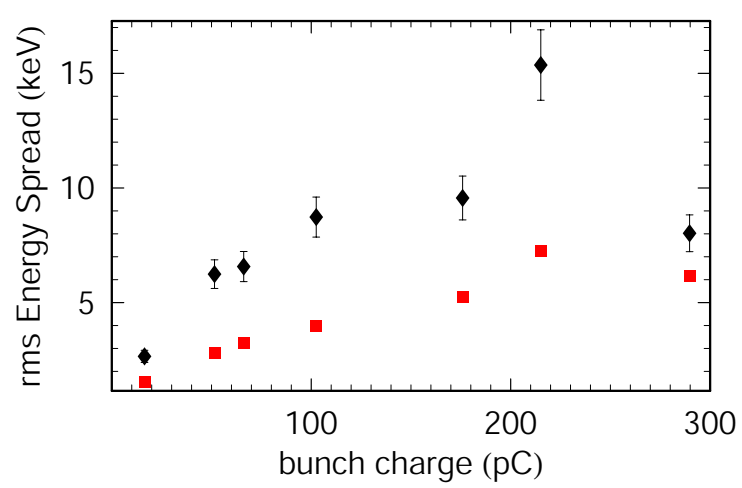

Figure 5: Effective uncorrelated energy spread measured in the GTF (black diamond) and the corresponding theoretical value (red square).

cases, the experimental values are somewhat larger than the analytical estimations. This can be understood because the electron bunch continues to evolve nonlinearly in the gun as well as in the subsequent accelerator sections.

\section{REFERENCES}

[1] M. Hüning and H. Schlarb, Proceedings of PAC2003, 2074 (2003).

[2] D. Dowell et al., Nucl. Instrum. Methods A 507, 331 (2003).

[3] H. Loos et al., Nucl. Instrum. Methods A 528, 189 (2004).

[4] M. Borland et al., Nucl. Instrum. Methods A 483, 268 (2002).

[5] E. Saldin, E. Schneidmiller, and M. Yurkov, Nucl. Instrum. Methods A 528, 355 (2004).

[6] Z. Huang et al., Phys. Rev. ST-AB 7074401 (2004).

[7] M. Cornacchia, and P. Emma, Phys. Rev. ST-AB 5, 084001 (2002).

[8] T. Shaftan, and L.-H. Yu, Phys. Rev. E 71, 046501 (2005).

[9] R. Helm, and R. Miller, in: Linear Accelerators, eds. P.M. Lapostolle and A.L. Septier (North-Holland, 1969), p. 115.

[10] Z. Li, private communication.

[11] K.-J. Kim, Nucl. Instrum. Methods A 275, 201 (1989).

[12] J. Billen, LANL Report LA-UR-96-1835 (1996).

[13] C. Limborg et al., Proceedings of PAC2003, 3216 (2003). 